\title{
GExpLine: A Tool for Supporting Experiment Composition*
}

\author{
Daniel de Oliveira ${ }^{1}$, Eduardo Ogasawara ${ }^{1}$, Fernando Seabra ${ }^{1}$, Vítor Silva ${ }^{1}$, \\ Leonardo Murta $^{2}$, and Marta Mattoso ${ }^{1}$ \\ ${ }^{1}$ COPPE, Federal University of Rio de Janeiro, Rio de Janeiro, Brazil \\ ${ }^{2}$ Fluminense Federal University, Niterói, Brazil \\ \{danielc, ogasawara, fernando_seabra, silva, marta\}@cos.ufrj.br, \\ leomurta@ic.uff.br
}

\begin{abstract}
Scientific experiments present several advantages when modeled at high abstraction levels, independent from Scientific Workflow Management System (SWfMS) specification languages. For example, the scientist can define the scientific hypothesis in terms of algorithms and methods. Then, this high level experiment can be mapped into different scientific workflow instances. These instances can be executed by a SWfMS and take advantage of its provenance records. However, each workflow execution is often treated by the SWfMS as independent instances. There are no tools that allow modeling the conceptual experiment and linking it to the diverse workflow execution instances. This work presents GExpLine, a tool for supporting experiment composition through provenance. In an analogy to software development, it can be seen as a CASE tool while a SWfMS can be seen as an IDE. It provides a conceptual representation of the scientific experiment and automatically associates workflow executions with the concept of experiment. By using prospective provenance from the experiment, GExpLine generates corresponding workflows that can be executed by SWfMS. This paper also presents a real experiment use case that reinforces the importance of GExpLine and its prospective provenance support.
\end{abstract}

\section{Introduction}

Scientific workflows are a prominent solution to model scientific experiments [1]. One fundamental problem is representing and tracking the composition process. Composing a scientific workflow involves many trials. Scientists usually execute different tasks during composition, such as: establishing the logical sequence of activities, planning variations that have to be explored, and defining the types of input and output data for each activity. All these actions are taken before using a SWfMS and are not registered.

Since scientists need to draw conclusions from a scientific experiment. During the experiment life cycle, many different workflow executions need to be evaluated by choosing different parameters, alternative data sets, programs, or even algorithms and

* This work was partially sponsored by CNPq and CAPES. 
methods [2]. Additionally, some activities may be optional in the workflow, i.e., scientists should explore the experiment by choosing to use (or not) a particular activity in order to model the entire workflow in a suitable way for the experiment being conducted. The exploration of all these different workflows represents trials that are performed to evaluate the initial hypothesis. Nevertheless, existing approaches are not conceived to relate two or more workflow trials as part of a single experiment. For instance, a SWfMS such as VisTrails [3] offers a framework for controlling workflow versions, which represent several modifications of the same executable workflow over the time. In VisTrails, different versions of an executable workflow are related and represented in an evolution version tree. Although these versions share common characteristics, they do not necessarily represent alternatives for the same experiment. For example, a new version of a workflow is generated by simply removing a specific activity from the workflow. The reason for this removal could be a bug fix or the exploration of a different type of result for the same experiment. Unless some annotation is used to represent which actions are allowed to be performed over a workflow (e.g., removing some specific activities), the decision regarding removing or choosing activities can be extremely error prone and counterproductive.

These several composition tasks should be registered in addition to producing an executable workflow. These composition tasks represent a significant amount of effort for the scientists. Representing a workflow in hierarchical abstract levels can help the process of analyzing the experiment, but this kind of representation is an open issue [4]. A conceptual workflow is a workflow where scientists define what they want to do without specifying how to do it.

Representing a workflow at hierarchical levels of abstraction presents several advantages. For example, it is possible to add semantics to the executable level by registering the main algorithms and software that originated the concrete workflow. It is also possible to group different concrete workflows as alternative (similar) solutions to one experiment. Thus, provenance from workflow trials is registered as part of one single experiment. Workflow reuse and generation of executable workflows from conceptual representations become simpler than browsing executable workflow repositories or querying workflow provenance databases.

GExpLine is a tool for supporting experiment composition. GExpLine is complementary to existing SWfMS, thus offering extra representation layers to be coupled to the existing workflow systems. Its goal is to provide a high abstraction level representation environment for scientists to model their scientific experiments and to automatically generate corresponding executable workflows in pre-defined SWfMS specification languages.

Provenance data is a key concept used to link the experiment of GExpLine to the SWfMS workflow executions. GExpLine provides mechanisms to gather prospective provenance data from the experiment definition. This prospective provenance data is related to the retrospective provenance data produced by the SWfMS. In addition, by coupling GExpLine to workflow ontologies, the experiment representation is boosted with ontology concepts, such as algorithms or methods inherent to scientific experiments, and the provenance may also be analyzed based on these concepts. The GExpLine representation is based on the concept of experiment lines [2].

The use case presented in this paper is based on a real experiment of the deep water oil exploitation domain to reinforce GExpLine's representation of experiments at 
higher abstraction levels, supporting workflow composition and the derivation [2] to different SWfMS with prospective provenance support. This paper is organized in four sections besides this introduction. Section 2 presents GExpLine architectural features. Section 3 presents details of the use case that demonstrates how to model a real experiment of deep water oil exploitation using GExpLine. Section 4 brings the related work and Section 5 concludes the paper.

\section{GExpLine}

The concept of experiment lines [2] is an innovative approach to represent a scientific experiment. It is based on the successful concept of software product lines [5]. An experiment line may be defined as a conceptual workflow that is capable to derive multiple workflows at the concrete level. It is a flow of activities where each activity behaves like an independent component [6]. When an activity of the flow can be implemented by any activity from a list of alternative activities, it is called a variation point. It means that there is more than one alternative program, algorithm or method to implement the variation point. Also, when an abstract activity can be suppressed when deriving a concrete workflow, in order to represent a different type of result or analysis, and not due to its incorrectness, it is defined as an optional activity. On the other hand, a mandatory activity is an activity that must be present in all derived concrete workflows. Experiment lines follow the optional, mandatory, and variant activities. In addition, GExpLine presents a powerful configuration management mechanism that allows the versioning of workflow elements to model the experiment.

The GExpLine tool is based on five main components: (i) Experiment Line Modeler: designs experiment lines, (ii) Derivation: derives concrete workflows based on abstract ones. The derivation process is based on the concept of cartridges, i.e. when scientists are deriving their conceptual workflows (represented in our object model) into concrete ones they have to choose one cartridge in a set of available cartridges, where each cartridge of this set generates concrete workflows for different representation language and also in XPDL [7] which is agnostic from SWfMS, (iii) Import: imports concrete workflows from Kepler [8], Taverna [9], and VisTrails [3]. This import process is also based on the concept of cartridges. There is a different cartridge implemented for each type of workflow language, and (iv) Version Control: controls versions of abstract/concrete workflows [10]. (v) Query: queries prospective provenance data using abstract/concrete information. In the current version, provenance queries are executed directly in SQL using DBMS functionalities. The following section explains in detail the GExpLine conceptual model that allows scientists to extract prospective provenance data from the modeled experiments.

\section{GExpLine Prospective Provenance Model}

The GExpLine prospective provenance model defines the experiment, abstract and concrete workflows, workflow components such as ports and relations, version identification and organization, as well as operations for retrieving existing versions and constructing new versions [10]. The GExpLine prospective provenance model is 
composed by three main parts: (i) workflow classes, in which workflow concepts are represented; and (ii) version classes, which represent the way that versions are organized. Figure 1 presents the GExpLine prospective provenance model as UML class diagram. Classes were colored differently since they represent different perspectives on the model. The white classes, which are Experiment, Workflow, Activity, Relationship, Port, AbstractActivity, ConcreteActivity, Derivation and MetaArtifact represent the workflow classes, which is actually the workflow meta-model. Finally, light gray classes, which are Version, Transaction, ConfigurationItem, User, and Project, represent the version classes. Workflow classes represent, in configuration management terminology [11], the product space. The version classes are called, in configuration management terminology, the version space [10]. The linking between the product space and version space is defined by the VersionedElement class, which acts as an interface between both spaces. All classes that are part of the workflow meta-model just need to inherit from the VersionedElement class to be versioned and managed by the configuration management mechanism.

A workflow (class Workflow) is composed of activities (class Activity) and relationships (class Relationship). The class Workflow may be specialized into conceptual abstract workflows (class AbstractWorkflow) and concrete workflows (class ConcreteWorkflow). An activity in a workflow has input and output ports (class Port). The relationship between activities is a directed edge that establishes the dependency between activities and also defines the workflow activity chaining. In addition, the class Activity presents a self-relationship that indicates variability, i.e., the choices that scientists make when modeling a workflow. There are two specializations for the Activity class. The AbstractActivity class represents activities modeled in the abstract level while ConcreteActivity represents activities modeled in the executable level. Both AbstractActivity and ConcreteActivity inherit from the class Activity. Each Activity that is part of a Workflow produces and consumes a specific MetaArtifact. A MetaArtifact is a type of artifact in a prospective provenance model. A generated artifact obtained during workflow execution is actually an instance of a MetaArtifact. The derivations performed by scientists are registered in the class Derivation. This way, the activities and workflows derived are registered and provide important information for future backtracking information.

It is possible to relate the GExpLine workflow meta-model with Open Provenance Model (OPM) [12] nodes. For example, the class MetaArtifact is mapped to the Artifact OPM node. Indeed, a MetaArtifact is a conceptual representation of an artifact node in OPM. The class Activity is mapped to the Process OPM node, since both of them are performed on artifacts. The class User is mapped to the Agent OPM node. This way it is possible to discover which entity composed and derived the activity. The composition and derivation actions can be mapped to the OPM Role, which in this case are compose and derive, since they designate an artifact's or agent's function in a process.

The version space is decoupled from the product space, allowing both spaces to evolve independently. The version space is composed by six main classes: ConfigurationItem, Version, Transaction, User, VersionedElement, and Project. Each configuration item (class ConfigurationItem) is composed by versions (class Version). Each version has relationships to the next and previous versions, which can be null for the first and last versions of a configuration item, respectively. A specific attribute differentiates versions 
that were deleted by the user. Additionally, there is a relationship to branched versions, which allows non-sequential development. Versions are queried or created by transactions (class Transaction) made by users (class User). Finally, versions have relationships to the versioned elements (interface VersionedElement).

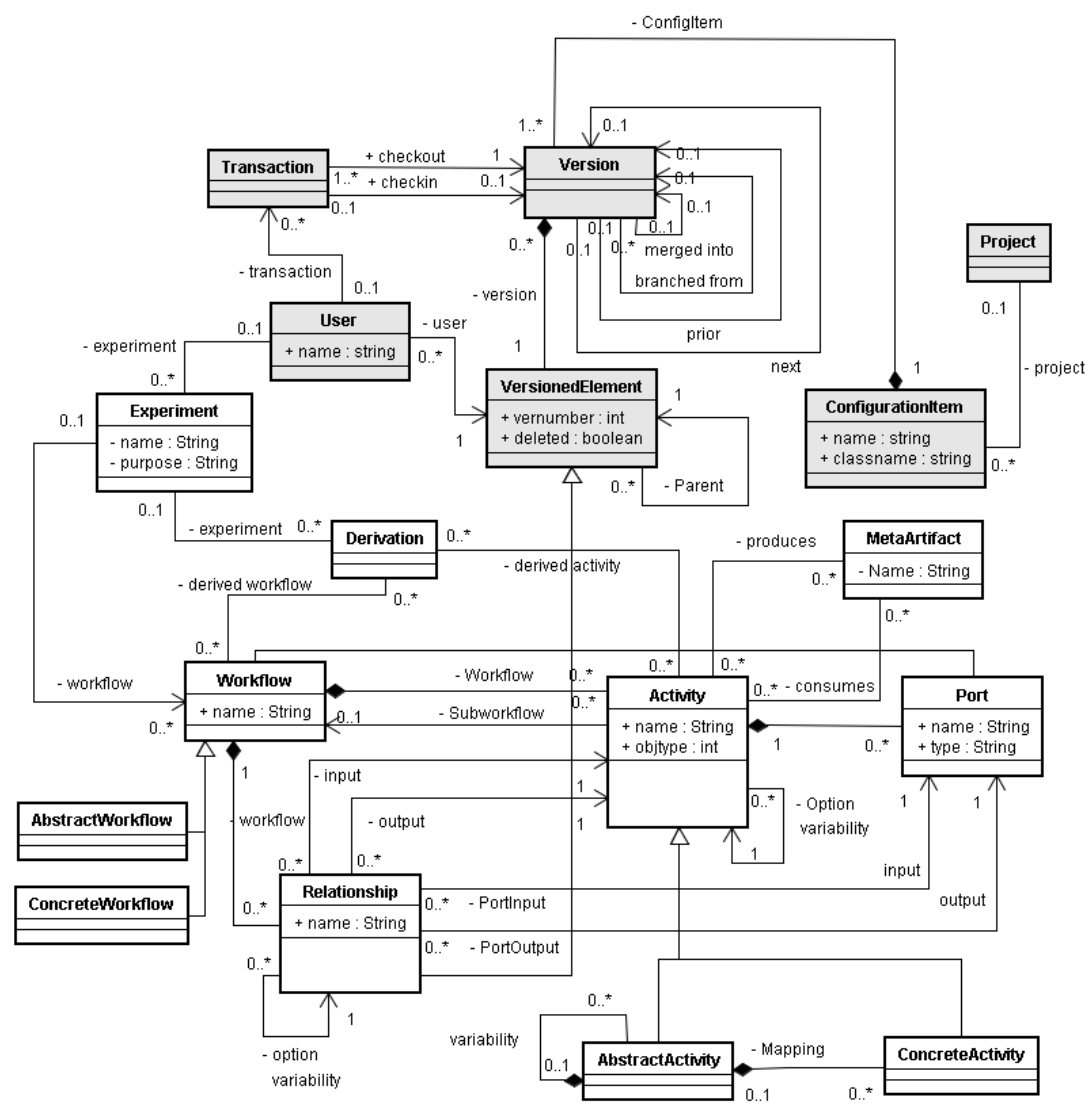

Fig. 1. GExpLine Prospective Provenance Model

VersionedElement interface is not exclusive for the version space. It is also part of the product space, since it connects the versioning space with the product space, as presented in Figure 1. Although a configuration item does not know more than the type and the hierarchy of one of the versioned element in the product space, it is used to retrieve the product information. Also, from the project (class Project), which is a representation of a compound object, it is possible to obtain the configuration item that is actually associated with the whole workflow. The conceptual model of Figure 1 was instantiated in a relational schema in the PostgreSQL DBMS and it is used as the prospective provenance schema for GExpLine. While using the information presented in this model, scientists are able to extract and analyze provenance data and 
draw important conclusions about the entire experiment. The next section presents a real experiment of the deep water oil exploitation scientific workflow modeled in GExpLine.

\section{Modeling a Deep Water Oil Exploitation Experiment}

The scientific experiment used in this GExpLine demonstration is based on the Deep Water Oil Exploitation Domain. Although the experiment is more complex and with many alternatives, for sake of simplicity, we present a small fraction of the experiment, which is composed by four conceptual activities that are sequentially connected: (i) Pre-Processing (implemented by PreProc program); (ii) Intermediate Processing (implemented by SigProc program), (iii) Structural Analysis (implemented by StruCAD or S-Analyst programs), and (iv) Generation of tension histogram (implemented by Histogram program). In this experiment, the fourth activity of the flow is optional, which means that it can be suppressed in some derived workflows, and the third activity is a variation point, which means that scientists have alternatives (StruCAD and S-Analyst) as presented in Figure 2. When using a SWfMS directly, these two workflows from Figure 2 would be managed independently by the SWfMS. Querying provenance corresponding to all executions of the same experiment line would require a significant effort through the SWfMS provenance support. It is not simple to gather and combine the provenance results of these specific executions.

Based on the experiment definition, the initial step is to set up the versioning mechanism in GExpLine. The scientist must create a workspace for this new experiment line. A workspace in the GExpLine context is an environment where scientists are able to model their experiment lines, isolated from the outside world, i.e., isolated from each other's work. The scientist is able to choose an experiment line version to work with if there is any committed version available.

The second step to be performed is to model the experiment line. It may be created from scratch (top-down) or by importing multiple concrete workflows and generalizing them (bottom-up). The experiment line is modeled just once and scientists may derive or make annotations any time after that.

From the modeled experiment line, scientists may conduct experiments by deriving concrete workflows associated to that experiment. In this demonstration, the first concrete workflow is composed by the programs PreProc, SigProc, StruCAD, and Histogram. Let us suppose that the scientists were not satisfied with the obtained results, and want to experiment a different method or algorithm. GExpLine can derive an alternative concrete workflow. In this second trial, the optional activity was suppressed and the concrete workflow has three activities, represented by the programs PreProc, SigProc, and S-Analyst. This change is part of the experimentation process and all this information is gathered and stored in the prospective provenance schema of GExpLine.

It is important to notice that the experiment line composition is highly dependent on the expertise of senior scientists and is a knowledge intense activity. However, using an existing experiment line to derive workflows is a less error prone activity and can be performed even by novice scientists. This occurs due to the effective use of composition rules (already adopted in software product lines) [13]. The essence of 
experiment lines is the systematic and efficient composition of experiments. This composition is made by chaining a series of programs in a coherent manner. However, not all programs are compatible. Composition rules are commonly used to define the legal combinations of programs in an experiment line. In addition to domain constraints, there are low level implementation constraints that must also be satisfied.

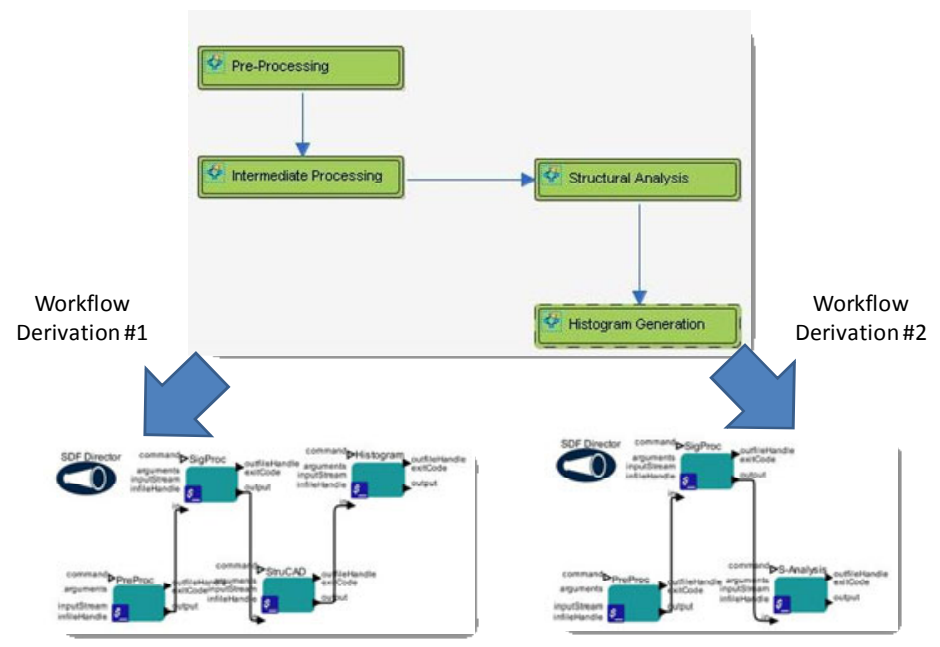

Fig. 2. Derivation in GExpLine

The demonstration ends with queries on prospective provenance data related to the experiment line using DBMS features. This query service allows analyzing information about concrete workflows associated with one experiment. Using the provenance query directly from the SWfMS, the user would have to gather the set of independent workflow executions corresponding to the experiment line. Let us take the example explained in this subsection. There are many generated concrete workflows for the same experiment. Particularly, the activity Structural Analysis has two different programs that implement it (StruCAD and S-Analyst) where each one produced different artifacts. If scientists want to discover which workflow produces the "tension amplitude histogram", using existing SWfMS, scientists are not able to discover the available workflows in the provenance schema (retrospective provenance is not available since the query is prior to execution). A demonstration video can be found at http://gexp.nacad.ufrj.br/news/gexpline-demo-ipaw.

\section{Related Work}

We have analyzed the literature and SWfMS support for workflow representation in different abstraction levels. Current SWfMS are restricted to one workflow execution at a time. In this way, the knowledge of which activities can be linked to each other is still tacit, since there is no conceptual representation of the experiment as happens in 
GExpLine. Currently, projects in many SWfMS force scientists to redefine, almost from scratch, scientific workflows previously developed by other scientists, incurring in the same composition trial and error. This occurs due to the absence of a systematic approach for composition and lack of a conceptual workflow representation.

Ludäscher et al. [14] present an approach to relieve the scientists from directly designing executable workflows. It represents an abstract workflow based on directed acyclic graphs. It proposes to use database mediation techniques that automatically map abstract workflow activities into executable ones. This mapping is powerful and independent of SWfMS. However, this approach does not add represent optionalities or variabilities that are important concepts for scientific experiments. Using those concepts, scientists are able to identify similar workflows that share a common ancestor.

The task specification language (TSL) [15] is an initiative to represent scientific workflows in different abstraction levels. The TSL approach is coupled to the VIEW [16] SWfMS, but it does not represent alternative activities and it does not concern about representing the concept of scientific experiment.

\section{Conclusion}

GExpLine allows scientists to model their experiments in a high abstraction level, improving the management of the scientific experiment. GExpLine provides features to associate different concrete workflows to a single conceptual definition of the experiment. This experiment to concrete workflow mapping is fundamental for scientific experiment management. It produces conceptual prospective provenance data for analysis together with provenance data from SWfMS concrete workflow instances. This prospective provenance data is thus complementary to the SWfMS provenance data. Ongoing work includes developing a lightweight interface for querying provenance data and incorporating composition rules in GExpLine.

\section{References}

[1] Taylor, I.J., Deelman, E., Gannon, D.B., Shields, M. (eds.): Workflows for e-Science: Scientific Workflows for Grids, 1st edn. Springer, Heidelberg (2007)

[2] Ogasawara, E., Paulino, C., Murta, L., Werner, C., Mattoso, M.: Experiment Line: Software Reuse in Scientific Workflows. In: 21th SSDBM, New Orleans, LA, pp. 264-272 (2009)

[3] Callahan, S.P., Freire, J., Santos, E., Scheidegger, C.E., Silva, C.T., Vo, H.T.: VisTrails: visualization meets data management. In: Proc. SIGMOD 2006, USA, pp. 745-747 (2006)

[4] Shoshani, A.: The Scientific Data Management Center: Providing Technologies for Large Scale Scientific Exploration. Scientific and Statistical Database Management, 1-2 (2009)

[5] Northrop, L.: SEI's software product line tenets. IEEE Software 19(4), 32-40 (2002)

[6] Szyperski, C.: Component Software: Beyond Object-Oriented Programming. AddisonWesley Professional, Reading (1997)

[7] I. WfMC, Binding, WfMC Standards, WFMC-TC-1023 (2009), http: / /www.wfmc.org (2000) 
[8] Altintas, I., Berkley, C., Jaeger, E., Jones, M., Ludascher, B., Mock, S.: Kepler: an extensible system for design and execution of scientific workflows. In: SSDBM, Greece, pp. 423-424 (2004)

[9] Hull, D., Wolstencroft, K., Stevens, R., Goble, C., Pocock, M.R., Li, P., Oinn, T.: Taverna: a tool for building and running workflows of services. Nucleic Acids Research 34(Web Server issue), 729-732 (2006)

[10] Ogasawara, E., Rangel, P., Murta, L., Werner, C., Mattoso, M.: Comparison and Versioning of Scientific Workflows. In: CVSM 2009, Vancouver, Canada, pp. 25-30 (2009)

[11] Conradi, R., Westfechtel, B.: Version Models for Software Configuration Management. ACM Computing Surveys 30(2), 232-282 (1998)

[12] Moreau, L., Freire, J., Futrelle, J., McGrath, R., Myers, J., Paulson, P.: The Open Provenance Model: An Overview. In: Provenance and Annotation of Data and Processes, pp. 323-326 (2008)

[13] Garg, A., Critchlow, M., Chen, P., Westhuizen, C.V.D., Hoek, A.V.D.: An Environment for Managing Evolving Product Line Architectures. In: Proceedings of the International Conference on Software Maintenance, pp. 358-366 (2003)

[14] Ludascher, B., Altintas, I., Gupta, A.: Compiling abstract scientific workflows into web service workflows. In: Proceedings of the 15th International Conference on Scientific and Statistical Database Management, Cambridge, MA, pp. 251-254 (2003)

[15] Lin, C., Lu, S., Fei, X., Pai, D., Hua, J.: A Task Abstraction and Mapping Approach to the Shimming Problem in Scientific Workflows. In: Proc. Services 2009, pp. 284-291 (2009)

[16] Lin, C., Lu, S., Lai, Z., Chebotko, A., Fei, X., Hua, J., Fotouhi, F.: Service-Oriented Architecture for VIEW: A Visual Scientific Workflow Management System. In: Services, pp. 335-342 (2008) 